\title{
Generating the Vertex Sets with some Distance Parameter Properties in Caterpillar Graphs
}

\author{
Shreedevi V. Shindhe \\ Dept of Computer Science \\ Karnatak University, \\ Dharwad, India
}

\author{
Ishwar. B \\ Dept of Computer Science \\ Karnatak University, \\ Dharwad, India
}

\author{
Marriswamy. $\mathrm{R}$ \\ Research Scholar \\ Dept of Computer Science \\ Karnatak University, \\ Dharwad, India
}

\begin{abstract}
In this paper the vertices of caterpillar tree $T$ are viewed with different approach and categorized into the sets $D, R$ and $X$, based on the distance parameters i.e., diameter and radius. The distance parameters have been presented with some set theory views. Here $D$ is the set of diametral vertices, $R$ is the set of central vertices and $X$ is the set of vertices which are neither central nor peripheral. Then, $|D|+|R|+|X|=n$. The cardinality of these sets has some property and helps to specify the basic characters of caterpillar tree. A linear complexity algorithm is also designed to generate these sets.
\end{abstract}

Keywords:

Diameter, Radius, Set representation, Caterpillar.

\section{BASIC DEFINITIONS:}

The distance $d(u, v)$ between two vertices $u$ and $v$ in a graph $G$ is the length of a shortest path between them.

The eccentricity $e(v)$ of a vertex $v$ in a graph $G$ is the maximum distance from $v$ to any other vertex. Therefore $e(v)=\max \{d(u, v): u \in V\}$. The diameter $\operatorname{diam}(G)$ of a graph $G$ is the maximum eccentricity over all vertices in a graph; and the radius $\operatorname{rad}(G)$, the minimum. Trivially, $\operatorname{diam}(G) \leq 2 \operatorname{rad}(G)$. Vertices with maximum eccentricity are called peripheral vertices or diametral vertices. Vertices of minimum eccentricity form the center and are called central vertices. A tree has at most two center vertices.

A caterpillar is a tree in which every graph vertex is on a central stalk or only one graph edge away from the stalk, in other words, removal of its endpoints leaves a path graph. A tree is a caterpillar iff all nodes of degree $\geq 3$ are surrounded by at most two nodes of degree two or greater. [1, 2]

\section{SET REPRESENTATION OF}

\section{VERTICES OF CATERPILLAR TREE:}

A graph $G$ is defined as the set of vertices and edges, i.e. $G=(V, E)$. Where $V$ is the set of vertices and $E$ is the set of edges. The set $V$ can be further divided into different subsets with some properties assigned to the vertices. For example, the subset of $V$ may be a set of dominating vertices or a set of independent vertices.

In this paper the vertices of caterpillar tree $T$ are viewed with different perspective and categorized into the sets $D, R$ and $X$, based on the distance parameters, diameter and radius. The distance parameters have been presented with some set theory views. The details and examples are as follows.
Let $T$ be a caterpillar tree with $n$ vertices. Every caterpillar tree has some diametral (peripheral) vertices and central vertices. In addition to these, some of the caterpillars also have the vertices which are neither central nor diametral. Let us call these vertices as $x$-vertices. Based on these three properties, vertex set of caterpillar can be categorised. The vertices of $T$ can be grouped into three sets $D, R$ and $X$ by considering the distance parameters diameter and radius.

Here $D$ is the set of all diametral vertices. It is represented as:

$$
D=\{v \mid v \text { is diametral vertex }\}
$$

$R$ is the set of all central vertices. Represented by:

$$
R=\{v \mid v \text { is central vertex }\}
$$

$X$ is the set of all vertices which are nether central nor diametral. We call these vertices as $x$-vertices. Represented by:

$$
X=\{v \mid v \text { is a } x-\text { vertex }\}
$$

The sum of cardinality of each set gives us total number of vertices in $T$. It is clearly seen that using the above sets we can write:

$$
|D|+|R|+|X|=n \quad \cdots \cdots \rightarrow
$$

From the Figure 1 , see that $\mathrm{n}=9$, and the set of vertices is $V=\{a, b, c, d, e, f, g, h, i\}$. Each of the vertex is given with its eccentricity. The diameter of the graph is 6 and radius is 3 . There are 3 diametral vertices, 1 central vertex and 5 vertices whose eccentricity nether equals to diameter nor equals to radius. Therefore $|D|=3,|R|=1$ and $|X|=5$. Expression 1 can be easily verified.

There are different conditions for these sets and their cardinality. These are dealt in detail in further sections.

\section{SOME BASIC PROPERTIES OF}

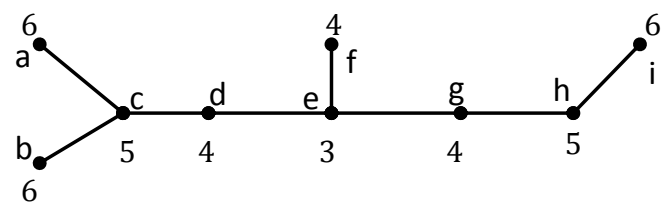

Figure 1: Caterpillar T 


\section{THESE SETS:}

\subsection{When $|D|=n$ or $|R|=n$ :}

When $|D|=n$ or $|R|=n$, it means that all the vertices are present either in the set $D$ or in the set $R$ i.e., diameter=radius. Hence $T$ is self centered. Of course in this case $|X|=0$. The only caterpillars that are self centered are $K_{1}$ and $K_{2}$. In Figure 2 the eccentricity of each vertex is specified.

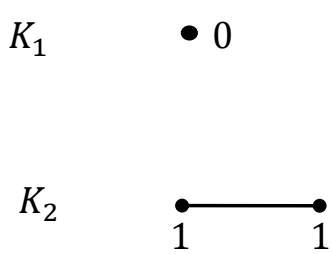

Figure 2: $K_{1}$ and $K_{2}$

\subsection{When $|R|=1$ or $|R|=2$ :}

When $|R|=1$, it means that there is only one central vertex, and hence the $T$ it central or unicentral. And while $|R|=2$, the $T$ is bicentral. In [2] it has been proved that a tree can have at the most two central vertices. So $1 \leq|R| \leq 2$. In this case $|D| \neq 0$ and $|X| \geq 0$.

\subsection{Vertices on the spine of caterpillar:}

The path induced by removing the end vertices of $T$ is called spine of caterpillar $[1,2]$. This path consists of central and $x$ vertices and $x$-vertices occur in pairs of same eccentric values. It can be seen in Figure 1 that the spine consists of path $c, d, e, g, h$ and these are the vertices with eccentric vertices $5,4,3,4,5$ respectively. Except the vertex $e$, the central vertex, the other vertices are the $x$-vertices.

From Figure 1, we observe that all the vertices on the spine, except the central vertices, belong to the set $X$. The eccentric values of vertices are such that it ranges from maximum eccentricity to minimum one, i.e. from diameter to radius. the number of paired spinal $x$-vertices can be computed by decrementing the diameter value by 1 till it is equal to radius. The same is represented as,

$$
\begin{gathered}
d, \underbrace{d-1, d-2, \ldots r+1, \quad r,}_{\text {spine vertices of unicentral caterpillar }} \quad r+1, \ldots d-1 \\
d, \underbrace{d-1, d-2, \ldots r+1, \quad r, r, \quad r+1, \ldots d-1}_{\text {spine vertices of bicentral caterpillar }}, d
\end{gathered}
$$

By ignoring central and diametral vertices we get the eccentricity of $x$-vertices on the spine,

$$
\underbrace{d-1, d-2, \ldots r+1, r+1, \ldots, d-2, d-1}_{x-\text { vertices of caterpillar }}
$$

The number of pairs of spinal $x$-vertices, $\mathrm{S}_{\mathrm{p}}$, (except Central vertices) can be obtained by,

$$
\begin{aligned}
S_{p} & =\sum_{i=r+1}^{d-1} 1 \\
& =d-1-(r+1)+1 \\
& =d-1-r-1+1 \\
\boldsymbol{S}_{\boldsymbol{p}} & =\boldsymbol{d}-\boldsymbol{r}-\mathbf{1}
\end{aligned}
$$

And, therefore the total number of spinal vertices including central vertices, can be obtained by the following expression,

$$
\text { No. of spine vertices }=2 S_{p}+|R| \cdots--(2)
$$

From the Figure 1, see that,

$$
d=6, r=3 \text { and }|R|=1
$$

$S_{p}=6-3-1=2$. Therefore,

no. of spine vertices $=2 * 2+1=5$.

\section{ALGORITHM TO GENERATE THE SETS $D, R$ AND $X$ :}

The sets of diametral vertices, central vertices and $\mathrm{x}$-vertices can be generated by the following method. First we need to find the eccentricity of each vertex and then have to find the maximum (diameter) and minimum (radius) of the eccentricities. The eccentricity of the vertices can be obtained by Floyd's algorithm [3], which takes $O\left(n^{3}\right)$ if we use matrix. Finding the maximum and minimum of the eccentricities can be done in $O(n)$ time by using divide and conquer Minimum and Maximum algorithm [4,5]. The following algorithm has been developed which determines the sets $D, R$ and $X$ and their cardinality in $O(n)$ time.

Let $T[n][n]$ be the input caterpillar tree, $n$ be the number of vertices of $T$. Let the arrays $D, R, X$ and $e[$ represent the sets $D, R, X$ and eccentricity of each vertex. Let $D_{\text {count }}, R_{\text {count }}$ and $X_{\text {count }}$ be the variables which will hold the cardinality of each set. Let the variables diam and rad hold the value of diameter and radius respectively.

Algorithm Generate_Sets(int $n$, int e[ ])

\{

$$
\begin{aligned}
& \text { int } D_{\text {count }}=0, R_{\text {count }}=0, X_{\text {count }}=0 \text {; } \\
& \text { for } i=1 \text { to } n \\
& \{ \\
& \text { if } \mathrm{e}[\mathrm{i}]==\text { diam } \\
& \{ \\
& D\left[D_{\text {count }}\right]=e[i] \\
& D_{\text {count }}++ \text {; } \\
& \text { \} } \\
& \text { else if e[i]=rad } \\
& \{ \\
& \mathrm{R}\left[R_{\text {count }}\right]=e[i] \\
& R_{\text {count }}++ \text {; } \\
& \text { \} } \\
& \text { else } \\
& \{ \\
& \mathrm{X}\left[X_{\text {count }}\right]=e[i]
\end{aligned}
$$


$X_{\text {count }}++$

\}

\}

\}

\section{Analysis of the algorithm:}

Since the algorithm has one for loop, the time complexity can be given as following:

$$
\begin{aligned}
T(n) & =\sum_{i=1}^{n} 1 . \\
& =n-1+1 \\
& =n .
\end{aligned}
$$

Therefore

$$
T(n)=O(n) .
$$

And hence the algorithm is linear.

\section{SOME PROPOSITIONS:}

Here are some propositions which can be easily observed by the given conditions. The caterpillar can be easily characterised as self centered and almost self centered by the following corollaries. [6,7 and 8].

Proposition 1: If $|R|=0$ AND $|X|=0$ AND $|D|=n$, then the caterpillar is self centered.

OR

If $|R|=n$ AND $|X|=0$ AND $|D|=0$, then the caterpillar is self centered.

Proposition 2: If $|D|=2$ AND $|R|=n-2$ AND $|X|=0$ then the caterpillar tree is almost self centered of type $(r+1, r)$.

Proposition 3: If $|D|=n-1$ AND $|R|=1$ AND $|X|=0$ then the caterpillar tree is almost self centered of type $(r, r+1)$.

\section{ACKNOWLEDGEMENTS}

My sincere regards to Dr. H. B. Walikar, Professor, Department of Computer Science, Karnatak University, Dharwad, for his valuable suggestions and corrections.

\section{CONCLUSION}

An effort has been made in this paper to characterise the vertices of caterpillar graph and generate the sets with some properties. This kind of generation of sets with some properties may help us further in dealing with problems with different views. We can refer the books $[9,10]$ for the details of set theory and further check with different set operations that can be performed on the sets $\mathrm{D}, \mathrm{R}$ and $\mathrm{X}$ and study the different properties exhibited by these operations on these sets.

\section{REFERENCES}

[1] Distance in graphs - Fred Buckley, Frank Harary, Addison-Wesley Pub. Co., (C1990 .

[2] Harary. F, Graph Theory, Addison Wesley.

[3] Coremen. T. H, Leiserson, C. E. Rivest R. L, and C Stein, Introduction to Algorithms, $2^{\text {nd }}$ Ed. MIT press, Cambridge, MA, 2001.

[4] Fundamentals of Computer Algorithms, E. Horowitz, S. Sahni, S. Rajasekharan, Universities Press, 2009.

[5] Data Structures, Algorithms, and Applications in $\mathrm{C}++$, Sartaj Sahni. McGraw-Hill Education, 1998.

[6] Shreedevi V. Shindhe, Ph. D thesis, "Algorithmic aspects of distance based parameters", Karnatak University, Dharwad, 2012

[7] H. B. Walikar, Shreedevi V. Shindhe, Ishwar Baidari DRI of a vertex, Self Centered Graphs and Almost Self Centered Graphs - An algorithmic approach,(152-156) International Journal of Computer Applications, Issue. 2, Vol. 4 August 2012.

[8] Sandi KLAVZAR, Kishori P. NARAYANKAR and H. B. WALIKAR -Almost Self-Centered Graphs, Acta Mathematica Sinica, English Series, Dec., 2011, Vol. 27, No. 12 , pp. 2343-2350.

[9] Discrete Mathematics and its Applications, Kenneth H. Rosen, $6^{\text {th }}$ Ed, Tata McGraw-Hill, 2007.

[10] Discrete Mathematical Structures- Kolman, Busby, Ross, $5^{\text {th }}$ Ed, PHI, 2000. 